\title{
Protective Effects of Carvacrol against Oxidative Stress Induced by Chronic Stress in Rat's Brain, Liver, and Kidney
}

\author{
Saeed Samarghandian, ${ }^{1}$ Tahereh Farkhondeh, ${ }^{2}$ Fariborz Samini, ${ }^{3}$ and Abasalt Borji ${ }^{1}$ \\ ${ }^{1}$ Department of Basic Medical Sciences, Neyshabur University of Medical Sciences, Neyshabur 14139-93186, Iran \\ ${ }^{2}$ Department of Immunogenetics, BuAli Research Institute, Mashhad University of Medical Sciences, Mashhad 9196773117, Iran \\ ${ }^{3}$ Department of Neurosurgery, Faculty of Medicine, Mashhad University of Medical Sciences, Mashhad 8564-917794, Iran
}

Correspondence should be addressed to Saeed Samarghandian; samarghandians@mums.ac.ir

Received 8 October 2015; Revised 25 December 2015; Accepted 30 December 2015

Academic Editor: John Voss

Copyright ( 2016 Saeed Samarghandian et al. This is an open access article distributed under the Creative Commons Attribution License, which permits unrestricted use, distribution, and reproduction in any medium, provided the original work is properly cited.

\begin{abstract}
Restraint stress may be associated with elevated free radicals, and thus, chronic exposure to oxidative stress may cause tissue damage. Several studies have reported that carvacrol (CAR) has a protective effect against oxidative stress. The present study was designed to investigate the protective effects of CAR on restraint stress induced oxidative stress damage in the brain, liver, and kidney. For chronic restraint stress, rats were kept in the restrainers for $6 \mathrm{~h}$ every day, for 21 consecutive days. The animals received systemic administrations of CAR daily for 21 days. To evaluate the changes of the oxidative stress parameters following restraint stress, the levels of malondialdehyde (MDA), reduced glutathione (GSH), superoxide dismutase (SOD), glutathione peroxidase (GPx), glutathione reductase (GR), and catalase (CAT) activities were measured in the brain, liver, and kidney. In the stressed animals that received vehicle, the MDA level was significantly higher $(P<0.001)$ and the levels of GSH and antioxidant enzymes were significantly lower than the nonstressed animals $(P<0.001)$. CAR ameliorated the changes in the stressed animals as compared with the control group $(P<0.001)$. This study indicates that CAR can prevent restraint stress induced oxidative damage.
\end{abstract}

\section{Introduction}

Plants products and their derivatives have been considered as an origin of therapeutic elements from ancient times. Today, there is basic research motivation in essential oils and extracts from different plant sources as potential antioxidant materials [1-5]. Carvacrol (5-isopropyl-2-methyl phenolCAR) is an ingredient of the essential oil obtained from Origanum hirtum, wild bergamot, pepperwort, and several other essential oils that possesses antioxidant and antimicrobial activities and a particular aroma which makes it an attractive component for certain types of foods [6]. CAR, or cymophenol, $\mathrm{C}_{6} \mathrm{H}_{3} \mathrm{CH}_{3}$, is a monoterpenoid phenol. It has a characteristic pungent, warm odor of oregano. The physicochemical characteristics of CAR and its chemical structure are presented in Table 1. CAR is considered safe for consumption and as a natural replacement of synthetic antioxidative food additives [6]. Several studies have shown that CAR has antioxidant, anti-inflammatory, antitumor, analgesic, antihepatotoxic, antimicrobial, and insecticidal activities [7]. CAR has strong antioxidant property and may be effective in prevention and inhibition of several diseases [8].

Oxidative stress is made by an imbalance between the generations of reactive oxygen species (ROS) and detoxifying the reactive intermediates via biological system's ability $[2,3$, 9]. Oxidative stress is a substantial mechanism that may be involved in the cytotoxicity induced by chronic stress [10]. Stress induced sympathetic stimulation causes an elevated respiration rate to generate more available oxygen for tissues. The increased metabolic rate also produces extra free radicals, leading to an imbalance between ROS generation and antioxidant system [10]. These free radical species result in oxidative damage to different molecules in cells, such as proteins, lipids, and nucleic acids [10].

It has been indicated that supplementation with natural antioxidants increases performance of the body organ during exposure to stressful environments $[4,11,12]$. CAR has 
TABLE 1: Physicochemical characteristics and molecular structure of carvacrol at $25^{\circ} \mathrm{C}$.

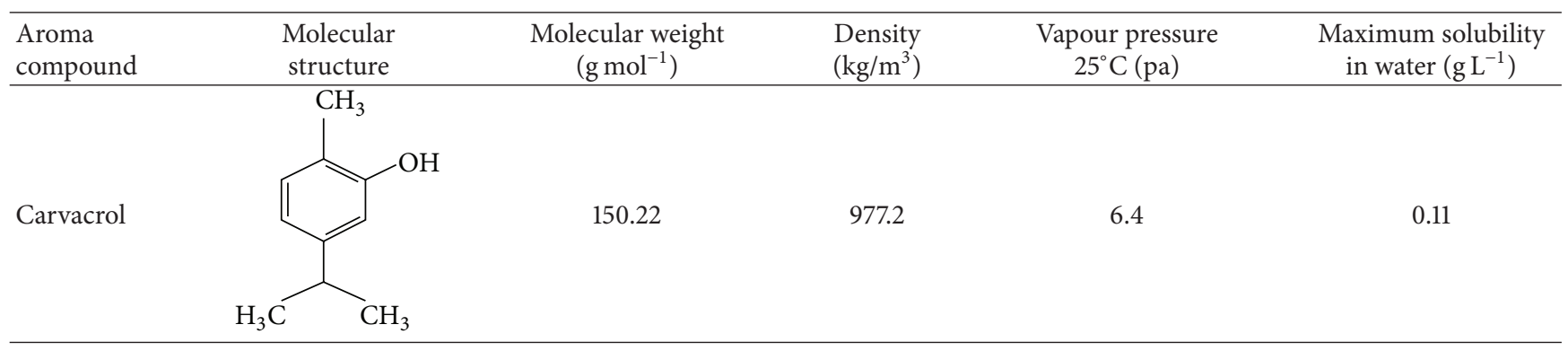

strong antioxidant activity [11]. CAR treatment significantly enhances the glutathione (GSH) level whereby the maintenance of GSH by CAR occurs basically due to removal of ROS through its radical scavenging effects [11]. It has been also illustrated that CAR raises total antioxidant capacity levels in cell cultures and animals [13]. Other investigations have identified that CAR protects against different pharmacological aspects, including anxiolytic-like, antitumor, antidepressant, antinociceptive, hypotensive, and antidiabetic activities [14, 15]. Since CAR is showed to have protective effect against the function of free radicals, we hypothesized that the administration of CAR might prevent chronic stress induced tissue damage through protection against oxidative stress.

Strong evidences have indicated the effect of various antioxidants on the chronic restraint or immobilizationinduced stress model [16]. Therefore, the present study was designed to investigate the effect of CAR on oxidative stressrelated changes in the brain, liver, and kidney of immobilization stress.

\section{Materials and Methods}

2.1. Reagents. All purified enzymes, coenzymes, substrates, standards, buffers, kits, and also carvacrol and other chemicals were purchased from Sigma-Aldrich Chemical (St. Louis, USA) and corticosterone ELIZA kit was purchased from Cusabio (Cusabio Biotech Co., Ltd.).

2.2. Animals. Wistar albino rats $(230 \pm 14.5 \mathrm{~g})$ were bred at the University Experimental Animal Care Centre. Animals were maintained under standard environmental conditions and had free access to standard rodent feed and water.

2.3. Study Design. Rats were randomly divided into eight experimental groups (8 rats per group) as follows: (1) vehicle (Veh) + no-stress (NS) (Veh-NS); (2) vehicle + stress (VehS), (3) CAR (20 mg/kg, IP) + no-stress (CAR20-NS), (4) CAR $(30 \mathrm{mg} / \mathrm{kg}$, IP) + no-stress (CAR30-NS); (5) CAR $(40 \mathrm{mg} / \mathrm{kg}, \mathrm{IP})+$ no-stress (CAR40-NS), (6) CAR $(20 \mathrm{mg} / \mathrm{kg}$, IP) + stress (CAR20-S), (7) CAR (30 mg/kg, IP) + stress (CAR30-S), and (8) CAR (40 mg/kg, IP) + stress (CAR40$\mathrm{S})$. Restraint stress was performed using a rodent restrainer made of Plexiglas that closely fit to the rats' body. For chronic restraint stress, rats were kept in the restrainers for $6 \mathrm{~h}$ per day for 21 consecutive days. The animals received systemic administrations of vehicle (dimethyl sulfoxide, DMSO) or CAR daily for 21 days [16]. At the end of the experimental period, animals were anesthetized by ether and blood was subsequently collected from the retroorbital sinus. Blood and sera were separated by centrifugation at $5000 \mathrm{rpm}$ for $5 \mathrm{~min}$ for corticosterone measurement. Then, brain, liver, and kidney were removed for measuring the oxidative stress markers. After the removal of tissues, they were washed in cold $0.9 \%$ saline and kept at $-70^{\circ} \mathrm{C}$ until they were used for preparation of homogenates with a homogenizer. Each tissue was finely minced and homogenized in $50 \mathrm{mM}$ phosphate buffer, $\mathrm{pH} 7.4$, and centrifuged at $10,000 \times \mathrm{g}$ for $15 \mathrm{~min}$ at $4^{\circ} \mathrm{C}$ (Beckman Refrigerated Ultracentrifuge). The homogenate and supernatant were used for the assays.

2.4. Corticosterone Evaluation. Under deep anesthesia, blood was collected from the retroorbital sinus of rats. Blood was allowed to clot and sera were separated using centrifugation at $5000 \mathrm{rpm}$ for $5 \mathrm{~min}$ and stored at $-80^{\circ} \mathrm{C}$ until use. Total serum level of corticosterone was measured by ELISA kits (CORT ELISA Kit CSB-E07014r).

2.5. Measurement of Lipid Peroxidation. Malondialdehyde (MDA) results from degradation of polyunsaturated lipids. The production of this substance is used as a biomarker to measure the level of lipid peroxidation. MDA reacts with thiobarbituric acid (TBA) as a thiobarbituric acid reactive substances (TBARS) to form a 1:2 MDA-TBA adduct, which is absorbed at $532 \mathrm{~nm}$. Thus, the quantity of TBARS is proportionate to the amount of MDA. Concentration of TBARS is determined according to a method of Uchiyama and Mihara. The concentration of TBARS was calculated using MDA standard curve and was expressed as nmol/mg of protein [17].

2.6. Estimation of GSH. GSH was measured by the method of Beutler et al. [18]. Briefly, to $0.1 \mathrm{~mL}$ of sample, $0.9 \mathrm{~mL}$ distilled water and $1.5 \mathrm{~mL}$ of precipitating reagent were added (3.34 g metaphosphoric acid, $0.4 \mathrm{~g}$ EDTA, and $60.0 \mathrm{~g}$ sodium chloride). Tubes were shaken and allowed to stand for $5 \mathrm{~min}$ at room temperature $\left(25 \pm 1^{\circ} \mathrm{C}\right)$. The mixture was centrifuged for $15 \mathrm{~min}$ at $4000 \mathrm{rpm}$ at $4^{\circ} \mathrm{C}$. In $1.0 \mathrm{~mL}$ supernatant, $4.0 \mathrm{~mL}$ of phosphate solution $(0.3 \mathrm{M}$ disodium hydrogen phosphate) and $0.5 \mathrm{~mL} 5$-50-dithiobis-(2-nitrobenzoic acid) (DTNB) ( $80 \mathrm{mg}$ in $1 \%$ sodium citrate) were added. The 
development of yellow color complex was read immediately at $412 \mathrm{~nm}$ on a spectrophotometer. A standard curve using GSH was prepared and GSH concentration in the experimental samples was extrapolated from the standard curve. GSH concentration was calculated and expressed as $\mu \mathrm{mol}$ of $\mathrm{GSH} / \mathrm{mg}$ protein.

2.7. Measurements of Enzymes. The activity of SOD was determined by the method of S. Marklund and G. Marklund [19], using inhibition of pyrogallol autoxidation at $\mathrm{pH} 8$. The specific activity of SOD is expressed as units per mg protein per minute. The activity of GPx was measured by the method of Paglia and Valentine [20]. GPx catalyses the oxidation of glutathione by cumene hydroperoxide. In the presence of glutathione reductase (GR) and NADPH the oxidized glutathione is immediately converted to the reduced form with a concomitant oxidation of NADPH to NADP. The decrease in absorbance at $340 \mathrm{~nm}$ is measured. GR catalyses the reduction of glutathione in the presence of NADPH, which is oxidized to NADP. The decrease in absorbance at $340 \mathrm{~nm}$ is measured. The levels of GPx and GR were expressed as $\mathrm{U} / \mathrm{mg}$ protein. CAT activity was assayed by $\mathrm{H}_{2} \mathrm{O}_{2}$ consumption, following Aebi's [21] method and modified by Pieper et al. [22].

2.8. Protein Estimation. Protein was estimated in subcellular fractions by the method of Bradford [23] using bovine serum albumin (BSA) as standard.

2.9. Statistical Analysis. All experiments were carried out at least in duplicate. Each group consisted of eight rats. Oneway analysis of variance (ANOVA) was performed and Tukey post hoc test was used for multiple comparisons. Statistical analyses were performed using the InStat 3.0 program. The results are expressed as mean \pm SEM. The results originated from analysis of serum. Differences of $P<0.05$ were considered significant.

\section{Results}

The levels of MDA, GSH, SOD, CAT, GPx, and GR in brain, liver, and kidney in the all groups are shown in Tables 2, 3, and 4. The MDA level of the Veh-S group in all tissues was significantly higher than those of Veh-NS and three CARNS groups $(P<0.001)$. The MDA level in the CAR40-S group in all tissues was significantly lower than those of the Veh-S group $(P<0.001)$. Our data showed that there was a significant difference in the MDA level in CAR40-S and CAR20-S groups in all tissues $(P<0.001)$. The MDA level of the CAR30-S in the brain and liver was significantly lower than CAR20-S group $(P<0.01)$. In addition, the MDA level of the CAR40-S in the brain was significantly lower than the CAR30-S group $(P<0.01)$.

The GSH level of the Veh-S group in all tissues was significantly lower than those of Veh-NS and three CARNS groups $(P<0.001$ for brain and kidney; $P<0.01$ for liver). The GSH level in the CAR40-S group in brain, liver, and kidney tissues was significantly higher than those of the

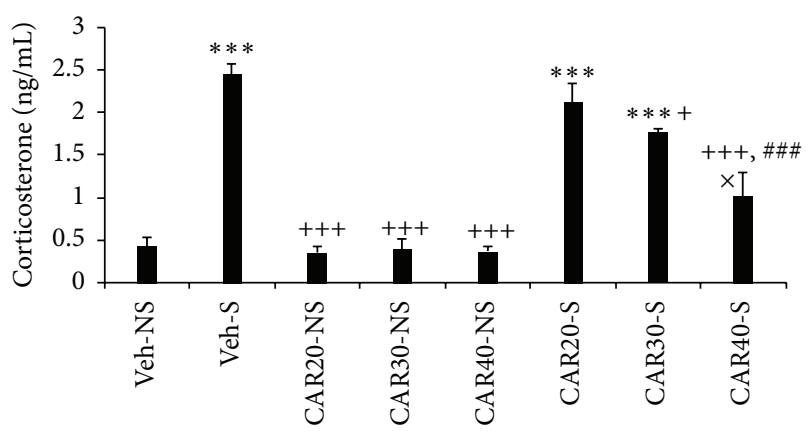

FIGURE 1: Effect of CAR on corticosterone levels in serum of immobilization stress and control groups ( $n=8$, for each group). Each measurement was done at least in triplicate and the values are the means \pm SEM for eight rats in each group. Significantly different from Veh-NS groups $\left({ }^{* * *} P<0.001\right)$. Significantly different from Veh-S groups $\left({ }^{+} P<0.05,{ }^{+++} P<0.001\right)$. Significant difference in CAR20-S versus CAR30-S and CAR40-S groups ( $\left.{ }^{\# \# ~} P<0.001\right)$. Significant difference in CAR30-S group versus CAR40-S group $\left({ }^{\times} P<0.05\right)$.

Veh-S group $(P<0.001, P<0.05$, and $P<0.001$, resp.). Our results illustrated that there was a significant difference between GSH level in the CAR40-S and the CAR20-S groups in the brain and kidney $(P<0.001)$. In addition, the GSH level of the CAR40-S in the brain was significantly higher than CAR30-S group $(P<0.01)$.

The activities of SOD $(P<0.001$ for brain and kidney; $P<0.05$ for liver $)$, GPx $(P<0.01$ for brain and kidney; $P<$ 0.001 for liver $)$, GR $(P<0.001$ for all tissues $)$, and CAT $(P<$ 0.001 for brain and kidney; $P<0.01$ for liver) in the Veh-S group in all tissues were significantly lower than those of VehNS and three CAR-NS groups. The SOD $(P<0.001$ for brain; $P<0.05$ for liver; $P<0.01$ for kidney $)$ GPx $(P<0.05$ for all tissues), GR $(P<0.05$ for all tissues $)$, and CAT $(P<0.001$ for brain; $P<0.05$ for liver; $P<0.01$ for kidney) activities in the CAR40-S group in all tissues were significantly higher than those of the Veh-S group. In addition, the SOD and CAT activities in the CAR30-S group in brain were significantly higher than those of the Veh-S group $(P<0.01)$. Present data indicated that there was a significant difference between the SOD ( $P<0.001$ for brain, $P<0.05$ for kidney), GPx $(P<$ 0.05 for kidney), and CAT $(P<0.001$ for brain, $P<0.01$ for kidney) activities in the CAR40-S and the CAR20-S groups in the brain and kidney. The significant difference was also observed in the CAT activity in the CAR40-S and the CAR30$\mathrm{S}$ groups in the kidney $(P<0.05)$.

The serum corticosterone level of the Veh-S group was significantly higher than those of Veh-NS and three CARNS groups $(P<0.001)$. The serum corticosterone level in the CAR30-S and CAR40-S groups was significantly lower than those of the Veh-S group $(P<0.05, P<$ 0.001 , resp.). The significant difference was observed in the serum corticosterone level in the CAR40-S and the CAR20$S$ and CAR30-S groups $(P<0.001, P<0.05$, resp. $)$ (Figure 1). 
TABLE 2: Effect of CAR on MDA (nmol/mgp), GSH ( $\mu \mathrm{mol} / \mathrm{mgp})$, SOD (U/mgp), GPx (U/mgp), GR (U/mgp), and CAT (U/mgp) levels in brain of immobilization stress and control groups ( $n=8$, for each group).

\begin{tabular}{lcccccc}
\hline Brain & MDA & GSH & SOD & GPx & GR & CAT \\
\hline Veh-NS & $2.68 \pm 0.12$ & $8.11 \pm 0.40$ & $3.12 \pm 0.10$ & $1.03 \pm 0.15$ & $0.85 \pm 0.11$ & $5.01 \pm 0.35$ \\
Veh-S & $6.28 \pm 0.24^{* * *}$ & $3.67 \pm 0.15^{* * *}$ & $0.98 \pm 0.13^{* * *}$ & $0.37 \pm 0.10^{* *}$ & $0.29 \pm 0.12^{* *}$ & $2.47 \pm 0.11^{* * *}$ \\
CAR20-NS & $2.23 \pm 0.18^{+++}$ & $8.01 \pm 0.39^{+++}$ & $3.06 \pm 0.16^{+++}$ & $0.96 \pm 0.13^{+}$ & $0.93 \pm 0.08^{+++}$ & $4.87 \pm 0.18^{+++}$ \\
CAR30-NS & $2.41 \pm 0.21^{+++}$ & $7.89 \pm 0.45^{+++}$ & $2.92 \pm 0.20^{+++}$ & $1.11 \pm 0.12^{+++}$ & $0.87 \pm 0.13^{++}$ & $5.10 \pm 0.15^{+++}$ \\
CAR40-NS & $2.36 \pm 0.17^{+++}$ & $7.95 \pm 0.48^{+++}$ & $3.23 \pm 0.15^{+++}$ & $1.17 \pm 0.10^{+++}$ & $0.90 \pm 0.10^{++}$ & $5.07 \pm 0.21^{+++}$ \\
CAR20-S & $5.13 \pm 0.25^{* * *++}$ & $4.02 \pm 0.22^{* * *}$ & $1.35 \pm 0.28^{* * *}$ & $0.55 \pm 0.05$ & $0.43 \pm 0.09$ & $2.93 \pm 0.20^{* * *}$ \\
CAR30-S & $3.98 \pm 0.11^{* * *++\# \#}$ & $5.18 \pm 0.29^{* * *}$ & $2.07 \pm 0.19^{* *++}$ & $0.74 \pm 0.09$ & $0.52 \pm 0.10$ & $3.72 \pm 0.13^{++* *}$ \\
CAR40-S & $2.87 \pm 0.19^{+++\# \#+\times}$ & $7.26 \pm 0.30^{+++\# \#+\times}$ & $2.85 \pm 0.24^{++\# \#}$ & $0.96 \pm 0.14^{+}$ & $0.74 \pm 0.05^{+}$ & $4.57 \pm 0.26^{+++\# \#}$ \\
\hline
\end{tabular}

Each measurement was done at least in triplicate and the values are the means \pm SEM for eight rats in each group.

Significantly different from Veh-NS groups $\left({ }^{* *} P<0.01,{ }^{* * *} P<0.001\right)$.

Significantly different from Veh-S groups $\left({ }^{+} P<0.05,{ }^{++} P<0.05\right.$, and $\left.{ }^{+++} P<0.001\right)$.

Significant difference in CAR20-S versus CAR30-S and CAR40-S groups $\left({ }^{\# \#} P<0.01,{ }^{\# \# \#} P<0.001\right)$.

Significant difference in CAR30-S group versus CAR40-S group $\left({ }^{\times \times} P<0.01\right)$.

TABLE 3: Effect of CAR on MDA (nmol/mgp), GSH ( $\mu \mathrm{mol} / \mathrm{mgp})$, SOD (U/mgp), GPx (U/mgp), GR (U/mgp), and CAT (U/mgp) levels in liver of immobilization stress and control groups $(n=8$, for each group).

\begin{tabular}{|c|c|c|c|c|c|c|}
\hline Liver & MDA & GSH & SOD & GPx & GR & CAT \\
\hline Veh-NS & $1.14 \pm 0.25$ & $10.09 \pm 1.08$ & $5.39 \pm 0.65$ & $1.04 \pm 0.09$ & $0.67 \pm 0.11$ & $6.09 \pm 0.24$ \\
\hline Veh-S & $3.97 \pm 0.16^{* * *}$ & $4.56 \pm 0.76^{* *}$ & $2.73 \pm 0.39^{*}$ & $0.45 \pm 0.10^{* * *}$ & $0.24 \pm 0.09^{* *}$ & $2.98 \pm 0.44^{* *}$ \\
\hline CAR20-NS & $0.98 \pm 0.10^{+++}$ & $9.81 \pm 0.90^{++}$ & $5.09 \pm 0.78^{+}$ & $1.01 \pm 0.02^{++}$ & $0.71 \pm 0.03^{++}$ & $5.87 \pm 0.76^{++}$ \\
\hline CAR30-NS & $1.06 \pm 0.19^{+++}$ & $10.16 \pm 1.06^{++}$ & $5.12 \pm 0.44^{+}$ & $0.89 \pm 0.06^{+}$ & $0.60 \pm 0.05^{+}$ & $5.67 \pm 0.81^{+}$ \\
\hline CAR40-NS & $1.20 \pm 0.21^{+++}$ & $10.00 \pm 0.95^{++}$ & $7.23 \pm 0.27^{+}$ & $1.12 \pm 0.14^{+++}$ & $0.58 \pm 0.07^{++}$ & $6.12 \pm 0.51^{++}$ \\
\hline CAR20-S & $3.01 \pm 0.11^{* * *++}$ & $6.12 \pm 1.11$ & $3.01 \pm 0.32^{*}$ & $0.58 \pm 0.03^{*}$ & $0.42 \pm 0.01$ & $3.72 \pm 0.38$ \\
\hline CAR30-S & $2.20 \pm 0.14^{* * *+++\# \#}$ & $7.46 \pm 0.50$ & $4.12 \pm 0.50$ & $0.69 \pm 0.07$ & $0.52 \pm 0.13$ & $4.53 \pm 0.53$ \\
\hline CAR40-S & $1.58 \pm 0.15^{+++\# \# \#}$ & $8.96 \pm 1.09^{+}$ & $5.06 \pm 0.21^{+}$ & $0.91 \pm 0.13^{+}$ & $0.61 \pm 0.08^{+}$ & $5.71 \pm 0.32^{+}$ \\
\hline
\end{tabular}

Each measurement was done at least in triplicate and the values are the means \pm SEM for eight rats in each group.

Significantly different from Veh-NS groups $\left({ }^{*} P<0.05,{ }^{* *} P<0.01\right.$, and $\left.{ }^{* * *} P<0.001\right)$.

Significantly different from Veh-S groups $\left({ }^{+} P<0.05,{ }^{++} P<0.05\right.$, and $\left.{ }^{+++} P<0.001\right)$.

Significant difference in CAR20-S group versus CAR20-S group $\left({ }^{\# \#} P<0.01,{ }^{\# \#} P<0.001\right)$.

\section{Discussion}

The present study indicates that chronic restraint stress induces oxidative stress in the brain, liver, and kidney and this oxidative stress damage in the tissues ameliorated by CAR treatment. Present data shows that CAR is effective against oxidative damage induced by chronic stress in the main organs. In this investigation MDA, marker for lipid per oxidation, exhibits the oxidative damage and the reduction of antioxidants. The levels of antioxidants including GSH, SOD, GPx, GR, and CAT were evaluated in the brain, liver, and kidney of rats exposed to restraint stress to determine the antioxidative potential of CAR. In the untreated control animals exposed to restraint stress, there was a considerable increase in the brain, liver, and kidney MDA levels, proposing stress induced lipid peroxidation. Present data are in agreement with the previous observations of increased levels of lipid peroxides in the brain, liver, and kidney of rats exposed to restraint stress [24]. The increase in tissue MDA level in the untreated control animals exposed to restraint stress accompanied by significant decrease in the GSH, SOD, GPx, GR, and CAT levels showed the overproduction of free radicals during immobilization stress. Treatment of animals with CAR resulted in decrease in the tissue MDA level and increase in the GSH, SOD, GPx, GR, and CAT activities levels, in comparison to the untreated exposed animals. The GSH level exhibits an essential role in detoxification in the tissue [25-28]. In the present study, GSH level was decreased in tissues of stress groups [29]. Stress decreases the GSH level and leads to increased levels of ROS in rat tissues [28]. Strong evidence has been indicated that the enzymatic antioxidant defense system against hydrogen peroxide $\left(\mathrm{H}_{2} \mathrm{O}_{2}\right)$, which is the high toxic substance for tissues, is primarily mediated by the GSH system $[4,25]$. GSH, as a cofactor of glutathione peroxidase (GPx), exhibits an essential role in the cell defense system. GSH, a thiol compound, has antioxidant activity in cells and reduces $\mathrm{H}_{2} \mathrm{O}_{2}$ and organic peroxides formation during lipid peroxidation with formation of oxidized glutathione disulfide (GSSG) [30]. In a normal physiological situation, glutathione exists as the reduced form (GSH) in cell; however, GSH is changed into its oxidized form (GSSG) by glutathione reductase (GR) when cells are exposed to overproduction of free radicals $[2,3,31]$. The peroxidase/glutathione reductase redox cycle is responsible for the maintenance of proper 
TABLE 4: Effect of CAR on MDA (nmol/mgp), GSH ( $\mu \mathrm{mol} / \mathrm{mgp})$, SOD (U/mgp), GPx (U/mgp), GR (U/mgp), and CAT (U/mgp) levels in kidney of immobilization stress and control groups ( $n=8$, for each group).

\begin{tabular}{lcccccc}
\hline Kidney & MDA & GSH & SOD & GPx & GR & CAT \\
\hline Veh-NS & $1.79 \pm 0.33$ & $7.65 \pm 0.36$ & $4.12 \pm 0.27$ & $2.34 \pm 0.15$ & $1.88 \pm 0.07$ & $5.01 \pm 0.24$ \\
Veh-S & $4.08 \pm 0.29^{* * *}$ & $3.81 \pm 0.41^{* * *}$ & $2.51 \pm 0.11^{* * *}$ & $0.86 \pm 0.12^{* *}$ & $1.02 \pm 0.11^{* *}$ & $2.73 \pm 0.11^{* * *}$ \\
CAR20-NS & $1.67 \pm 0.21^{+++}$ & $7.29 \pm 0.28^{+++}$ & $4.01 \pm 0.14^{++}$ & $2.05 \pm 0.10$ & $1.68 \pm 0.23^{+}$ & $4.89 \pm 0.31^{+++}$ \\
CAR30-NS & $1.83 \pm 0.38^{+++}$ & $7.83 \pm 0.47^{+++}$ & $3.87 \pm 0.33^{++}$ & $2.11 \pm 0.23$ & $1.93 \pm 0.14^{+++}$ & $5.22 \pm 0.42^{+++}$ \\
CAR40-NS & $1.74 \pm 0.41^{+++}$ & $7.08 \pm 0.32^{+++}$ & $4.28 \pm 0.10^{+++}$ & $1.92 \pm 0.27^{+}$ & $2.03 \pm 0.10^{+++}$ & $5.10 \pm 0.23^{+++}$ \\
CAR20-S & $3.77 \pm 0.25$ & $4.65 \pm 0.12^{* * *}$ & $2.88 \pm 0.20$ & $1.02 \pm 0.48$ & $1.24 \pm 0.05$ & $2.65 \pm 0.27^{* * *}$ \\
CAR30-S & $2.90 \pm 0.17$ & $5.85 \pm 0.22^{* *++}$ & $3.43 \pm 0.45$ & $1.37 \pm 0.26$ & $1.36 \pm 0.17$ & $3.12 \pm 0.51^{* *}$ \\
CAR40-S & $1.93 \pm 0.46^{++\# \#}$ & $6.88 \pm 0.31^{++\# \#}$ & $4.05 \pm 0.19^{++\#}$ & $2.25 \pm 0.33^{+\#}$ & $1.72 \pm 0.16^{+}$ & $4.55 \pm 0.18^{++\#+*}$ \\
\hline
\end{tabular}

Each measurement was done at least in triplicate and the values are the means \pm SEM for eight rats in each group.

Significantly different from Veh-NS groups $\left({ }^{* *} P<0.01,{ }^{* * *} P<0.001\right)$.

Significantly different from Veh-S groups $\left({ }^{+} P<0.05,{ }^{++} P<0.05\right.$, and $\left.{ }^{+++} P<0.001\right)$.

Significant difference in CAR20-S versus CAR30-S and CAR40-S groups $\left({ }^{\#} P<0.05,{ }^{\# \#} P<0.01\right.$, and $\left.{ }^{\# \# \#} P<0.001\right)$.

Significant difference in CAR30-S group versus CAR40-S group $\left({ }^{\times} P<0.05\right)$.

GSH concentration [32]. Changes in the activity of GPx and GR can disturb the concentration of GSH level [32]. In this study, treatment with CAR ameliorated the oxidative stress induced decrease in the GSH, SOD, GPx, GR, and CAT levels. SOD is a main antioxidant enzyme for scavenging superoxide anion. The activity of SOD which decreased in rat liver, brain, and kidney $[29,33]$ during restraint stress constitutes an important defense system to clear up ROS in vivo.

Our results confirmed the previous studies so that restraint stress induces free radical production and decreases antioxidant enzyme activities. In the physiological aspects, stress induces oxygen free radicals generation mostly formed in mitochondria, peroxisomes, lysosomes, cytosol, and the plasma membrane in body [34]. However, in the biochemical view, an imbalance between ROS generation and its clearance by the antioxidant defense system in the body has been observed in brain, liver, and kidney damage $[28,35]$. The produced free radicals from cellular metabolic processes have long been involved in the cellular toxicity $[35,36]$. An immobilization stress response results in the overproduction of free radicals that leads to lipid peroxidation, particularly in cell membranes [37]. The lipid peroxidation can change membrane integrity and then leads to tissue damage $[10,36]$. Pervious findings demonstrated that antioxidant enzymes activity was lower in liver, brain, and kidney after immobilization stress in animals [38]. In addition, the MDA content increased in all tissues especially in brain [38]. Similarly, present study indicated that oxidative stress induces oxidative injury in brain, liver, and kidney via increasing the MDA level and decreasing the GSH and antioxidant enzymes activity. We also observed that CAR ameliorated these modifications.

In this study, serum corticosterone level was measured in the rats immediately after chronic stress. According to the present findings, chronic stress increased serum corticosterone and CAR significantly decreased corticosterone level. Glucocorticoids exert an essential role in chronic stress induced oxidative injury [39]. Glucocorticoids may enhance the tissue MDA in stressed rats and this is a direct relation between serum corticosterone and the liver MDA level $[39,40]$. Furthermore, raised levels of glucocorticoids during restraint stress may affect the animal antioxidant content [39]. The mechanisms illustrated above elaborated in tissue damage induced by oxidative stress in current study.

The present investigation indicates that the injurious effects of chronic stress ameliorated by CAR treatment, proposing a protective effect of these agents against chronic stress. CAR also prevents lipid peroxidation by inducing SOD, GPx, GR, and CAT. CAR efficiently scavenges free radicals such as peroxyl radicals, superoxide radicals, hydrogen peroxide, and nitric oxide [41, 42]. CAR exerts antioxidant effect both in vitro and in vivo and its antioxidant activity is attributed to the presence of hydroxyl group $\left(\mathrm{OH}^{\circ}\right)$ linked to aromatic ring $[43,44]$. The increased levels of lipid peroxidation products in plasma, liver, kidney, and liver and the decreased levels of enzymic and nonenzymic antioxidants in rat were restored to normalcy after CAR treatment. CAR treatment inhibited free radicals formation and lipid peroxidation levels, which further improves membrane fluidity. CAR has been found to act as a radical scavenger inhibiting lipid peroxidation in vivo and in vitro.

In conclusion, the present study shows that CAR can inhibit chronic stress induced oxidative damage of the brain, liver, and kidneys. Thus, CAR should be fruitful as new pharmacological agent for ameliorating chronic stress induced oxidative damage.

\section{Conflict of Interests}

The authors report no declarations of interests.

\section{Acknowledgment}

The authors would like to thank Research Affairs of Neyshabur University of Medical Sciences for financially supporting this work. 


\section{References}

[1] F. E. Koehn and G. T. Carter, "The evolving role of natural products in drug discovery," Nature Reviews Drug Discovery, vol. 4, no. 3, pp. 206-220, 2005.

[2] S. Samarghandian, M. Azimi-Nezhad, and F. Samini, "Preventive effect of safranal against oxidative damage in aged male rat brain," Experimental Animals, vol. 64, no. 1, pp. 65-71, 2015.

[3] S. Samarghandian, M. Azimi- Nezhad, F. Samini, and T. Farkhondeh, "Chrysin treatment improves diabetes and its complications in streptozotocin-induced diabetic rats," Canadian Journal of Physiology and Pharmacology, 2015.

[4] V. Gupta, S. S. Lahiri, S. Sultana, R. K. Tulsawani, and R. Kumar, "Anti-oxidative effect of Rhodiola imbricata root extract in rats during cold, hypoxia and restraint (C-H-R) exposure and poststress recovery," Food and Chemical Toxicology, vol. 48, no. 4, pp. 1019-1025, 2010.

[5] K. Pan, Q. Jiang, G. Liu, X. Miao, and D. Zhong, "Optimization extraction of Ganoderma lucidum polysaccharides and its immunity and antioxidant activities," International Journal of Biological Macromolecules, vol. 55, pp. 301-306, 2013.

[6] S. Burt, "Essential oils: their antibacterial properties and potential applications in foods-a review," International Journal of Food Microbiology, vol. 94, no. 3, pp. 223-253, 2004.

[7] M. Melusova, D. Slamenova, K. Kozics, S. Jantova, and E. Horvathova, "Carvacrol and rosemary essential oil manifest cytotoxic, DNA-protective and pro-apoptotic effect having no effect on DNA repair," Neoplasma, vol. 61, no. 6, pp. 690-699, 2014.

[8] W. Z. Liang and C. H. Lu, "Carvacrol-induced $\left[\mathrm{Ca}^{2+}\right]_{\mathrm{i}}$ rise and apoptosis in human glioblastoma cells," Life Sciences, vol. 90, no. 17-18, pp. 703-711, 2012.

[9] H. Sies, "Strategies of antioxidant defense," European Journal of Biochemistry, vol. 215, no. 2, pp. 213-219, 1993.

[10] B. Halliwell, "Free radicals, antioxidants, and human disease: curiosity, cause, or consequence?” The Lancet, vol. 344, no. 8924, pp. 721-724, 1994.

[11] R. Aeschbach, J. Löliger, B. C. Scott et al., "Antioxidant actions of thymol, carvacrol, 6-gingerol, zingerone and hydroxytyrosol," Food and Chemical Toxicology, vol. 32, no. 1, pp. 31-36, 1994.

[12] S. Samarghandian, A. Borji, S. K. Farahmand, R. Afshari, and S. Davoodi, "Crocus sativus L. (saffron) stigma aqueous extract induces apoptosis in alveolar human lung cancer cells through caspase-dependent pathways activation," BioMed Research International, vol. 2013, Article ID 417928, 12 pages, 2013.

[13] E. Aydin, H. Türkez, and M. S. Keleş, “The effect of carvacrol on healthy neurons and N2a cancer cells: some biochemical, anticancerogenicity and genotoxicity studies," Cytotechnology, vol. 66, no. 1, pp. 149-157, 2014.

[14] F. H. C. Melo, E. T. Venâncio, D. P. De Sousa et al., "Anxiolyticlike effect of Carvacrol (5-isopropyl-2-methylphenol) in mice: Involvement with GABAergic transmission," Fundamental and Clinical Pharmacology, vol. 24, no. 4, pp. 437-443, 2010.

[15] F. H. C. Melo, B. A. Moura, D. P. de Sousa et al., "Antidepressantlike effect of carvacrol (5-Isopropyl-2-methylphenol) in mice: involvement of dopaminergic system," Fundamental and Clinical Pharmacology, vol. 25, no. 3, pp. 362-367, 2011.

[16] I.-S. Hong, H.-Y. Lee, and H.-P. Kim, "Anti-oxidative effects of rooibos tea (Aspalathus linearis) on immobilization-induced oxidative stress in rat brain," PLoS ONE, vol. 9, no. 1, Article ID e87061, 2014.
[17] M. Uchiyama and M. Mihara, "Determination of malonaldehyde precursor in tissues by thiobarbituric acid test," Analytical Biochemistry, vol. 86, no. 1, pp. 271-278, 1978.

[18] E. Beutler, O. Duron, and B. M. Kelly, "Improved method for the determination of blood glutathione," Journal of Laboratory and Clinical Medicine, vol. 61, pp. 882-888, 1963.

[19] S. Marklund and G. Marklund, "Involvement of the superoxide anion radical in the autoxidation of pyrogallol and a convenient assay for superoxide dismutase," European Journal of Biochemistry, vol. 47, no. 3, pp. 469-474, 1974.

[20] D. E. Paglia and W. N. Valentine, "Studies on the quantitative and qualitative characterization of erythrocyte glutathione peroxidase," The Journal of Laboratory and Clinical Medicine, vol. 70, no. 1, pp. 158-169, 1967.

[21] H. Aebi, "Catalase in vitro," Methods in Enzymology, vol. 105, pp. 121-126, 1984.

[22] G. M. Pieper, M. Jordan, L. A. Dondlinger, M. B. Adams, and A. M. Roza, "Peroxidative stress in diabetic blood vessels. Reversal by pancreatic islet transplantation," Diabetes, vol. 44 , no. 8 , pp. 884-889, 1995.

[23] M. M. Bradford, "A rapid and sensitive method for the quantitation of microgram quantities of protein utilizing the principle of protein-dye binding," Analytical Biochemistry, vol. 72, no. 1-2, pp. 248-254, 1976.

[24] S. P. Singh, A. J. Janecki, S. K. Srivastava et al., "Membrane association of glutathione S-transferase mGSTA4-4, an enzyme that metabolizes lipid peroxidation products," The Journal of Biological Chemistry, vol. 277, no. 6, pp. 4232-4239, 2002.

[25] R. Dringen, "Metabolism and functions of glutathione in brain," Progress in Neurobiology, vol. 62, no. 6, pp. 649-671, 2000.

[26] R. Dringen, J. M. Gutterer, and J. Hirrlinger, "Glutathione metabolism in brain: metabolic interaction between astrocytes and neurons in the defense against reactive oxygen species," European Journal of Biochemistry, vol. 267, no. 16, pp. 4912-4916, 2000.

[27] J. W. Gawryluk, J.-F. Wang, A. C. Andreazza, L. Shao, and L. T. Young, "Decreased levels of glutathione, the major brain antioxidant, in post-mortem prefrontal cortex from patients with psychiatric disorders," International Journal of Neuropsychopharmacology, vol. 14, no. 1, pp. 123-130, 2011.

[28] Y. Tang and Z. Zhong, "Obtusifolin treatment improves hyperlipidemia and hyperglycemia: possible mechanism involving oxidative stress," Cell Biochemistry and Biophysics, vol. 70, no. 3, pp. 1751-1757, 2014.

[29] B. Ghadrdoost, A. A. Vafaei, A. Rashidy-Pour et al., "Protective effects of saffron extract and its active constituent crocin against oxidative stress and spatial learning and memory deficits induced by chronic stress in rats," European Journal of Pharmacology, vol. 667, no. 1-3, pp. 222-229, 2011.

[30] V. Parked and J. Piotrowsk, "Glutathione: its role in detoxication of reactive oxygen and environmental chemicals," Acta Poloniae Toxicologica, vol. 4, article 1, 1996.

[31] M. Arai, H. Imai, T. Koumura et al., "Mitochondrial phospholipid hydroperoxide glutathione peroxidase plays a major role in preventing oxidative injury to cells," The Journal of Biological Chemistry, vol. 274, no. 8, pp. 4924-4933, 1999.

[32] R. C. Strange, P. W. Jones, and A. A. Fryer, "Glutathione Stransferase: genetics and role in toxicology," Toxicology Letters, vol. 112-113, pp. 357-363, 2000.

[33] N. P. Sudheesh, T. A. Ajith, V. Ramnath, and K. K. Janardhanan, "Therapeutic potential of Ganoderma lucidum (Fr.) P. Karst. 
against the declined antioxidant status in the mitochondria of post-mitotic tissues of aged mice," Clinical Nutrition, vol. 29, no. 3, pp. 406-412, 2010.

[34] J. Djordjevic, A. Djordjevic, M. Adzic, A. Niciforovic, and M. B. Radojcic, "Chronic stress differentially affects antioxidant enzymes and modifies the acute stress response in liver of wistar rats," Physiological Research, vol. 59, no. 5, pp. 729-736, 2010.

[35] J. M. C. Gutteridge, "Lipid peroxidation and antioxidants as biomarkers of tissue damage," Clinical Chemistry, vol. 41, no. 12, part 2, pp. 1819-1828, 1995.

[36] S. Samarghandian, R. Afshari, and T. Farkhondeh, "Effect of long-term treatment of morphine on enzymes, oxidative stress indices and antioxidant status in male rat liver," International Journal of Clinical and Experimental Medicine, vol. 7, no. 5, pp. 1449-1453, 2014.

[37] E. Şahin and S. Gümüşlü, "Immobilization stress in rat tissues: alterations in protein oxidation, lipid peroxidation and antioxidant defense system," Comparative Biochemistry and Physiology C-Toxicology and Pharmacology, vol. 144, no. 4, pp. 342-347, 2007.

[38] A. R. Bandegi, A. Rashidy-Pour, A. A. Vafaei, and B. Ghadrdoost, "Protective effects of Crocus sativus L. extract and crocin against chronic-stress induced oxidative damage of brain, liver and kidneys in rats," Advanced Pharmaceutical Bulletin, vol. 4, pp. 493-499, 2014.

[39] C. D. Conrad, K. J. McLaughlin, J. S. Harman et al., "Chronic glucocorticoids increase hippocampal vulnerability to neurotoxicity under conditions that produce CA3 dendritic retraction but fail to impair spatial recognition memory," The Journal of Neuroscience, vol. 27, no. 31, pp. 8278-8285, 2007.

[40] T. Farkhondeh, S. Samarghandian, M. Azimin-Nezhad, and F. Samini, "Effect of chrysin on nociception in formalin test and serum levels of noradrenalin and corticosterone in rats," International Journal of Clinical and Experimental Medicine, vol. 8, no. 2, pp. 2465-2470, 2015.

[41] A. T. Hariri, S. A. Moallem, M. Mahmoudi, B. Memar, and H. Hosseinzadeh, "Sub-acute effects of diazinon on biochemical indices and specific biomarkers in rats: protective effects of crocin and safranal," Food and Chemical Toxicology, vol. 48, no. 10, pp. 2803-2808, 2010.

[42] R. Kohen and A. Nyska, "Oxidation of biological systems: oxidative stress phenomena, antioxidants, redox reactions, and methods for their quantification," Toxicologic Pathology, vol. 30, no. 6, pp. 620-650, 2002.

[43] B. Aristatile, A. K. S. Numair, A. H. A. Assaf, C. Veeramani, and K. V. Pugalendi, "Protective effect of carvacrol on oxidative stress and cellular DNA damage induced by UVB irradiation in human peripheral lymphocytes," Journal of Biochemical and Molecular Toxicology, vol. 29, no. 11, pp. 497-507, 2015.

[44] A. G. Guimarães, G. F. Oliveira, M. S. Melo et al., "Bioassayguided evaluation of antioxidant and antinociceptive activities of carvacrol," Basic and Clinical Pharmacology and Toxicology, vol. 107, no. 6, pp. 949-957, 2010. 

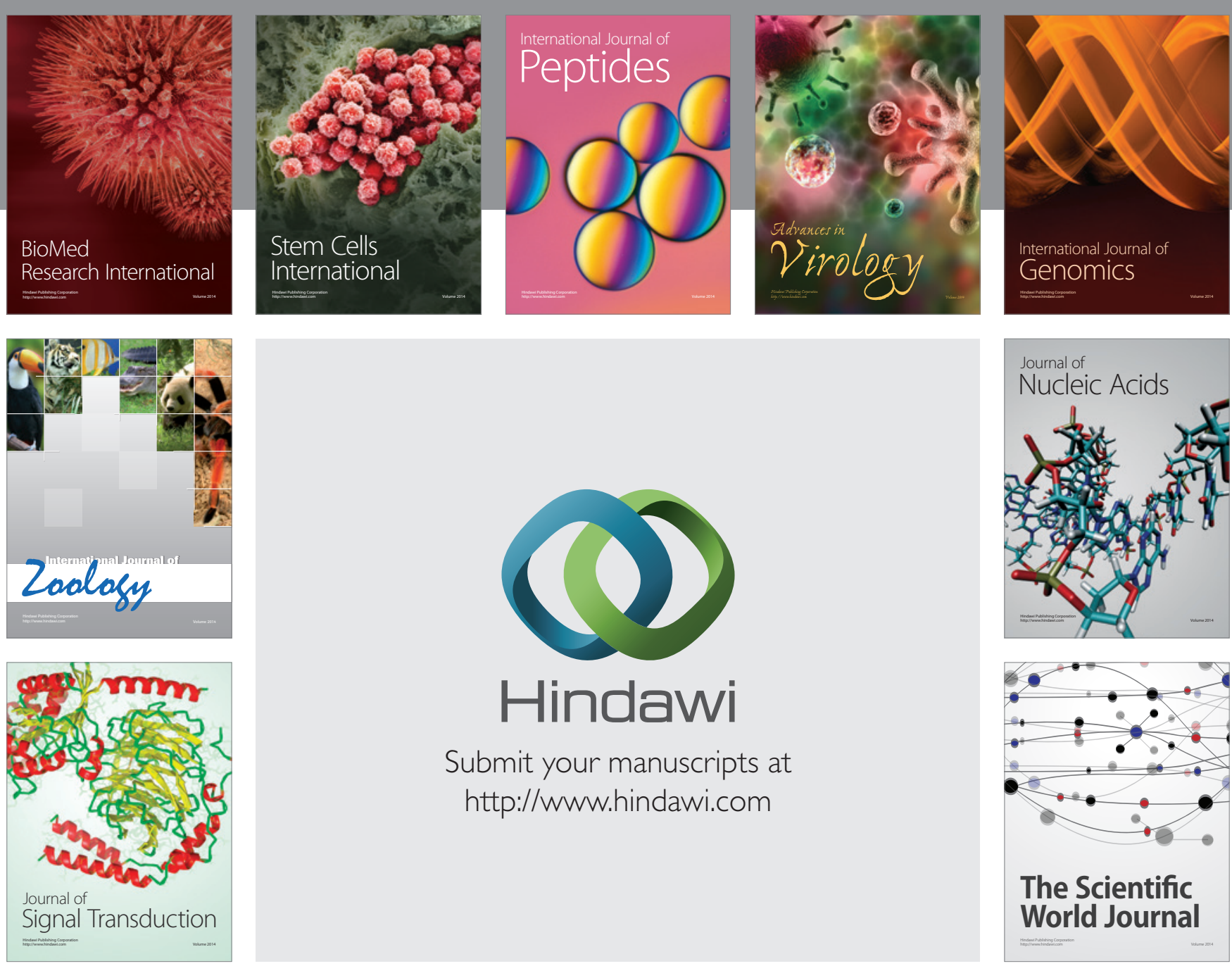

Submit your manuscripts at

http://www.hindawi.com
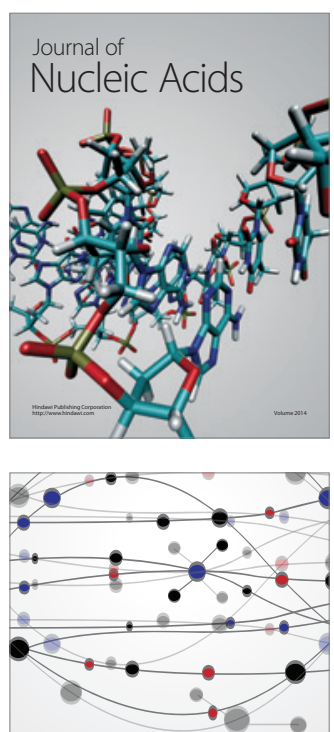

The Scientific World Journal
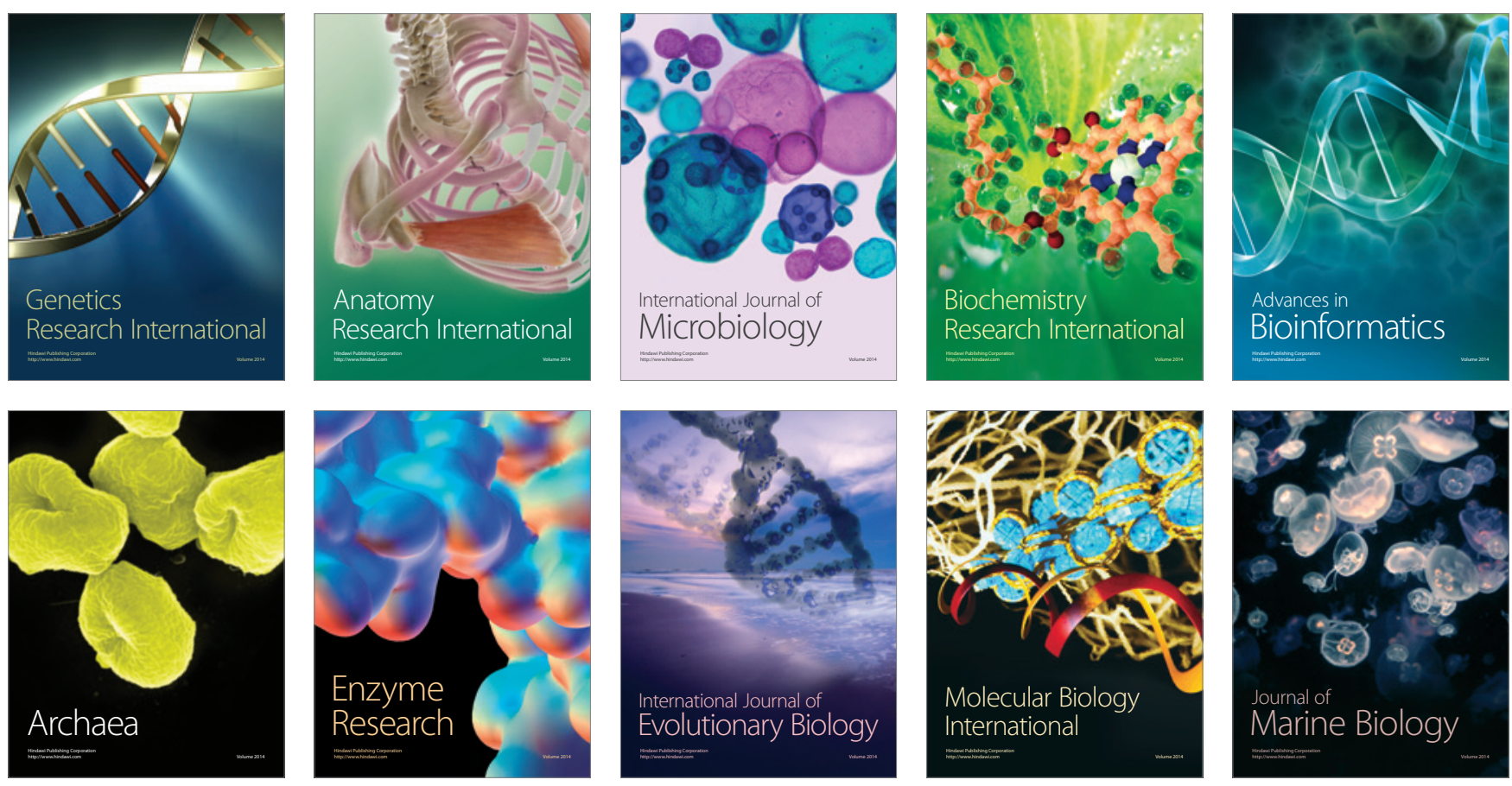